\title{
Multiple convex demarcation line for prediction of benign depressed gastric lesions in magnifying narrow-band imaging
}

(1) $\odot \ominus$

\author{
Authors

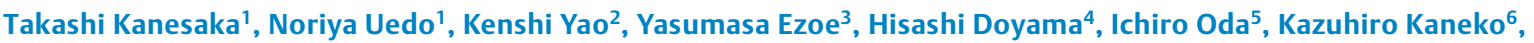 \\ Yoshiro Kawahara7, Chizu Yokoi ${ }^{8}$, Yasushi Sugiura ${ }^{9}$, Hideki Ishikawa ${ }^{10}$, Yoji Takeuchi ${ }^{1}$, Masamichi Arao ${ }^{1}$, Taro \\ Iwatsubo', Hiroyoshi Iwagami', Kenji Matsuno', Manabu Muto ${ }^{3}$, Yutaka Saito ${ }^{5}$, Yasuhiko Tomita ${ }^{11}$
}

Institutions

1 Department of Gastrointestinal Oncology, Osaka International Cancer Institute, Osaka, Japan

2 Department of Endoscopy, Fukuoka University Chikushi Hospital, Fukuoka, Japan

3 Department of Therapeutic Oncology, Graduate School of Medicine, Kyoto University, Kyoto, Japan

4 Department of Gastroenterology, Ishikawa Prefectural Central Hospital, Ishikawa, Japan

5 Endoscopy Division, National Cancer Center Hospital, Tokyo, Japan

6 Department of Gastroenterology, Endoscopy Division, National Cancer Center Hospital East, Chiba, Japan

7 Division of Endoscopy, Okayama University, Okayama, Japan

8 Endoscopy Division, National Center for Global Health and Medicine, Tokyo, Japan

9 Division of Gastroenterology and Hepatology, Kitano Hospital, Osaka, Japan

10 Department of Molecular Targeting Cancer Prevention, Kyoto Prefectural University of Medicine, Kyoto, Japan

11 Department of Pathology and Cytology, Osaka International Cancer Institute, Osaka, Japan

submitted 11.3.2017

accepted after revision 8.9.2017

Bibliography

DOI https://doi.org/10.1055/s-0043-121267 |

Endoscopy International Open 2018; 06: E145-E155

(c) Georg Thieme Verlag KG Stuttgart · New York

ISSN 2364-3722

Corresponding author

Takashi Kanesaka, Department of Gastrointestinal

Oncology, Osaka International Cancer Institute, 3-1-69,

Otemae, Chuo-ku, Osaka 541-8567, Japan

Fax: +81-6-6945-1900

kanesaka-ta@mc.pref.osaka.jp

\section{ABSTRACT}

Background and study aims With magnifying narrowband imaging (M-NBI) of the gastric mucosa, a characteristic demarcation line (DL) is occasionally found in non-cancerous depressed lesions. This DL forms multiple convex shapes along the edge of the epithelia of surrounding mucosa. We have termed this novel finding a multiple convex $\mathrm{DL}$ (MCDL). In this study, we clarified the prevalence of an MCDL in depressed gastric lesions detected in patients at high risk for gastric cancer and determined the diagnostic yield necessary to distinguish between cancer and non-cancer.

Patients and methods This was a post hoc analysis of a multicenter prospective trial. In total, 362 small $(\leq 10 \mathrm{~mm})$ depressed lesions were detected in 1353 patients. Presence or absence of a DL in target lesions was evaluated on M-NBI images. The proportion of MCDLs among lesions with a DL was evaluated.

Results Images of 347 lesions (39 cancerous and 308 noncancerous) were evaluable. A DL was present in 252/347 lesions $(73 \%)$. When the cutoff value for the proportion of MCDLs needed to distinguish non-cancer from cancer was set at two-thirds, an MCDL was observed in 86/252 lesions (34\%). In 86 lesions with an MCDL, 83 (97\%) were non-cancerous. The sensitivity, specificity, positive predictive value, and negative predictive value of an MCDL for non-cancerous lesions were $38 \%, 91 \%, 97 \%$, and $19 \%$, respectively.

Conclusions Presence of an MCDL had high specificity and positive predictive value for non-cancerous lesions. Evaluating the shape of the DL is useful for differentiation between cancer and non-cancerous lesions. 


\section{Introduction}

Endoscopic evaluation of lesion characteristics is the first step in diagnosis of gastric cancer, and subsequent biopsy is performed when the lesions are suspicious for neoplasia [1,2]. More than $60 \%$ of early gastric cancers show a depressed-type appearance $[3,4]$. However, in patients with atrophic gastritis, benign depressed lesions are more often detected during screening endoscopy [5]. Because the gross appearance of such lesions is similar, it is difficult to distinguish small early gastric cancer from benign depressed lesions by conventional white-light endoscopy. Although chromoendoscopy using indigo carmine may improve the diagnosis of early gastric cancer, no comparative study has proved the superiority of chromoendoscopy to white-light endoscopy. Accordingly, in everyday clinical practice, multiple forceps biopsies are usually performed to distinguish between early gastric cancer and small benign depressed lesions.

Narrow-band imaging (NBI) is an image-enhanced technology [6] that is generally used in combination with magnifying endoscopy in the diagnosis of gastric cancer. Magnifying NBI (M-NBI) allows for clearly visualization of the microsurface structure and microvascular architecture of the gastric mucosa [7, 8]. Yao et al. [8-11] indicated that an irregular microvascular pattern (IMVP) and/or irregular microsurface pattern with a demarcation line (DL) are the diagnostic criteria for early gastric cancer with M-NBI [vessel plus surface (VS) classification system]. The usefulness of M-NBI for the diagnosis of early gastric cancer was demonstrated by 2 multicenter prospective trials $[5,12]$. However, low inter-observer agreement regarding the endoscopic findings of M-NBI has also been reported as a problematic issue [13].

We recently noticed a characteristic finding of a DL that is observed mainly in non-cancerous depressed lesions in M-NBI. This DL can be clearly traced along the edge of the epithelia in the surrounding mucosa and forms multiple convex shapes on the inside ( $\mathbf{F i g}$. 1). We have coined this endoscopic finding a multiple convex DL (MCDL). If this finding is specific for noncancerous lesions, it will contribute to the differential diagnosis between cancer and non-cancerous lesions by M-NBI. In the present study, we aimed to clarify the prevalence of the MCDL in gastric depressed lesions detected by screening and the diagnostic performance of the MCDL in distinguishing between cancer and non-cancerous lesions.

\section{Patients and methods}

\section{Study design and participants}

This was a post hoc study using the data from a multicenter prospective trial in 9 hospitals in Japan (UMIN-CTR000001072) [5]. The protocol for the trial was approved by the Ethics Committee of the Kyoto University Graduate School of Medicine on February 14, 2008. The trial was conducted according to the Standards for the Reporting of Diagnostic Accuracy Studies (STARD) initiative and followed the Declaration of Helsinki [14]. Written informed consent was obtained from all patients. In this study, the participants and endoscopy system and set-

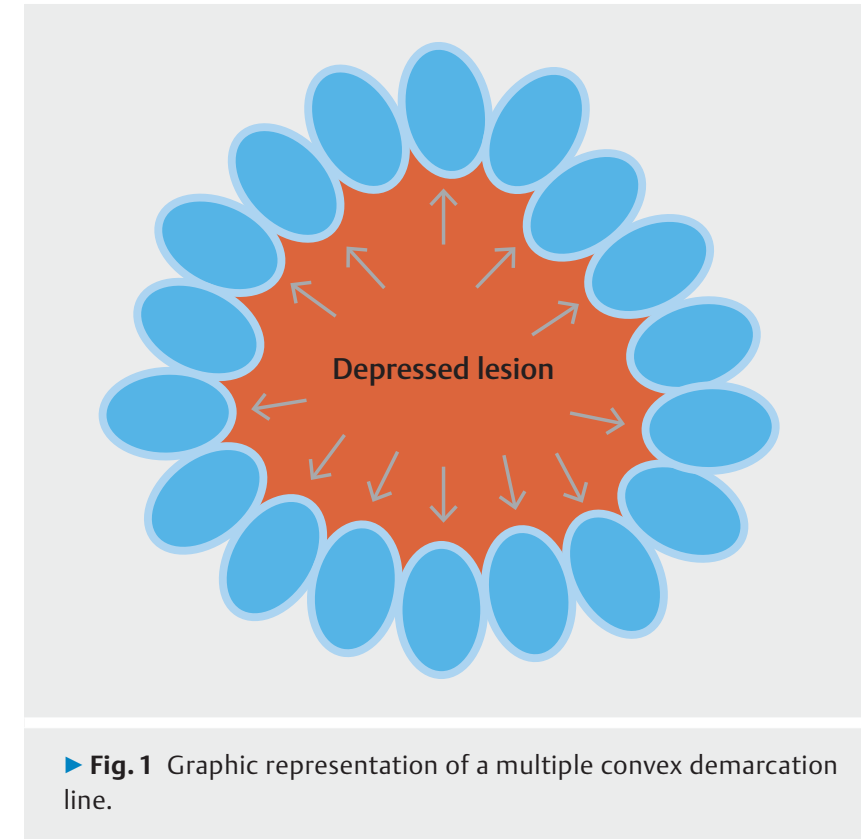

ting were taken from our previous report [15] because these were both post hoc analyses of the same prospective trial.

Patients aged $\geq 20$ years with concomitant gastric cancer or a history of endoscopic resection of gastric cancer were recruited from June 2008 to May 2010. Patients with a history of gastric resection, severe organ failure, and anticoagulant medication were excluded. In total, 1353 patients were enrolled and underwent endoscopic screening with conventional white-light endoscopy. The target lesions in 362 patients were defined as newly detected and undiagnosed depressed gastric lesions of $\leq 10 \mathrm{~mm}$ in diameter; because small, depressed cancers are difficult to distinguish from benign abnormalities such as inflammation. Only the first lesion detected in each patient was included for analysis to ensure independency of the analysis unit. Other detected lesions were examined according to protocols in ordinary clinical practice. At least 2 endoscopic images of the target lesions were recorded during diagnosis and stored in a computer image server. After accomplishing all endoscopic diagnoses, at least one biopsy specimen was obtained from the target lesion. Lesions that were diagnosed as cancer or suspicious of cancer were removed by endoscopic resection for final histological diagnosis. Three patients who did not follow the study protocol and 6 patients who missed biopsy were excluded; thus 353 patients were analyzed in the prospective trial ( Fig. 2).

Specimens obtained by biopsy or endoscopic resection were histologically evaluated using hematoxylin and eosin staining. The histological diagnosis was made according to the revised Vienna classification [16]. Categories 4 (noninvasive, highgrade neoplasia) and 5 (invasive neoplasia) were classified as cancer, and Categories 1 (negative for neoplasia), 2 (indefinite for neoplasia), and 3 (noninvasive, low-grade neoplasia) were classified as non-cancer. To confirm the histological diagnosis, endoscopic resection was performed for the lesions that were endoscopically suspected to be cancer. There were no cancer- 


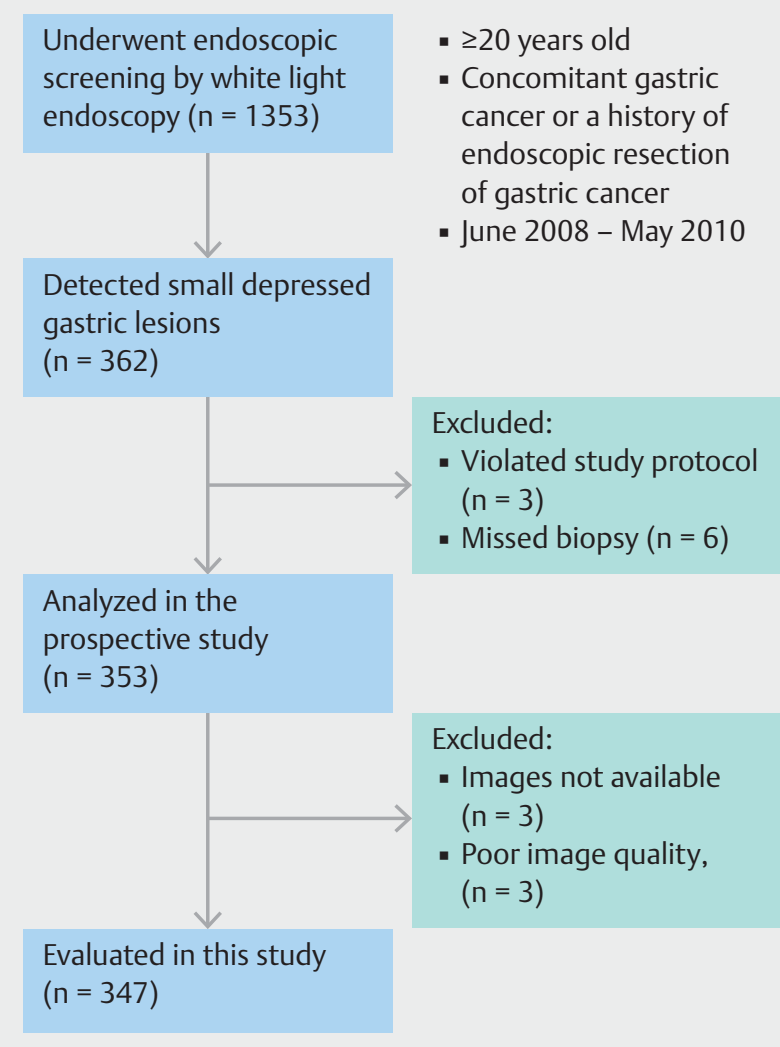

Fig. 2 Method for selection of samples in this study.

ous lesions that had disappeared at the time of endoscopic resection.

\section{Endoscopy system and setting}

The endoscopy system consisted of a video processor (CV260SL; Olympus Co., Tokyo, Japan) and a light source (CLV260SL; Olympus Co.) that worked in both the high-resolution white-light imaging and NBI modes. NBI illuminates narrowbanded short-wavelength light (400-430 and 525-555nm) to contrast the vascular architecture and surface structure of the superficial mucosa [6]. Magnifying endoscopy that can achieve a maximum 80-fold optical magnification was used (GIF-Q240Z, GIF-H260Z, and GIF-FQ260Z; Olympus Co.). A soft black hood (MB162 or MB46; Olympus Co.) was attached to the tip of the endoscope to enable the endoscopist to maintain an adequate distance for maximal magnification of the endoscopic image. The structural enhancement of the endoscopic video processor was set to B-mode level 4 or 6 for white-light endoscopy and to B-mode level 8 for M-NBI. The color mode was set at level 1.

\section{Evaluation of endoscopic findings}

In M-NBI, a DL was defined as a circumferential boundary between lesion and non-lesion areas that was discernible by an abrupt change in the microvessel and/or microsurface pattern of the mucosa [17]. We then defined a subtype of DL that fulfilled the following criteria for an MCDL: (1) a DL that could be
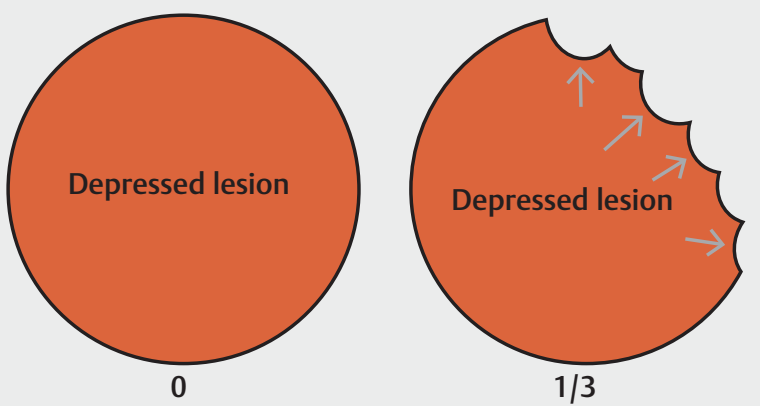

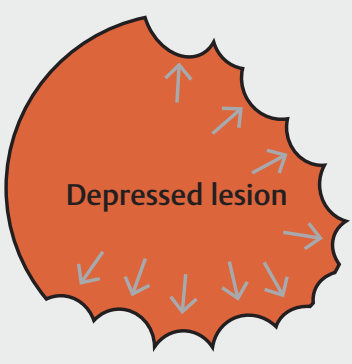

$2 / 3$

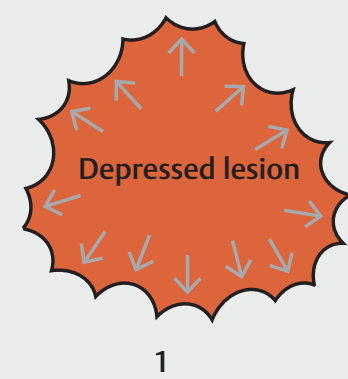

1
- Fig. 3 Visual analog scale for the graded proportion of MCDL among lesions with a DL.

clearly traced along the edge of the epithelia in the surrounding mucosa and (2) a DL that showed multiple convex shapes on the inside ( $\mathbf{F i g}$. 1).

$\mathrm{M}-\mathrm{NBI}$ images of all targeted lesions were collected from each participating hospital for evaluation of the aforementioned endoscopic findings. An experienced endoscopist (T.K., experience with $\geq 1,000$ cases involving M-NBI diagnosis), who was blinded to the histological findings, reviewed the recorded endoscopic images. First, the presence or absence of a DL was determined. The proportion of MCDLs among lesions with a DL was then determined. Using a visual analog scale ( $\mathbf{F i g . ~} \mathbf{3}$ ), this proportion was graded as $0,>0$ to $<1 / 3, \geq 1 / 3$ to $<2 / 3$, and $\geq 2 / 3$.

\section{Evaluation of reproducibility of endoscopic findings}

One experienced endoscopist (N.U., experience with $>1000$ cases involving $\mathrm{M}-\mathrm{NBI}$ diagnosis) and four less-experienced endoscopists (M. A., T.I., H.I. and K. M., each having experience with < 1000 cases involving M-NBI diagnosis) were invited to assess the diagnostic ability and inter-observer agreement of the endoscopic findings. They were asked to independently diagnose the presence or absence of a DL and the proportion of MCDLs. Before the assessment, the endoscopic findings and diagnostic criteria for both DL and MCDL were explained by the primary investigator (T.K.) using other sets of M-NBI images to control the diagnostic quality. 


\section{Evaluation of histological characteristics of non-cancerous lesions}

To clarify the histological characteristics of non-cancerous lesions with an MCDL, we reviewed the biopsy specimens from the participants enrolled in our institution. Mononuclear cell infiltration (inflammation), neutrophil infiltration (activity), glandular atrophy (atrophy), intestinal metaplasia, and Helicobacter pylori infection were evaluated and graded as normal, mild, moderate, or marked according to the visual analog scale in the updated Sydney system by one pathologist (Y.T.) who was unaware of the endoscopic findings [18].

\section{Statistical analysis}

The sensitivity, specificity, positive predictive value (PPV), negative predictive value (NPV), and accuracy of a DL for diagnosis of cancer were calculated. The optimal cutoff value for the proportion of MCDLs for distinguishing non-cancerous lesions from cancer was then determined by calculating the Youden's J index. For lesions with a DL, the sensitivity, specificity, PPV, NPV, and accuracy of an MCDL for non-cancerous lesions were calculated. The average values among the 6 endoscopists were also evaluated. The topographic distribution of lesions with an MCDL was also investigated to identify any association between the presence of an MCDL and lesion localization.

The $x^{2}$ test was used to evaluate associations of each endoscopic finding with cancer and localization of lesions with an MCDL. The Cochran-Armitage trend test was used to assess the association between endoscopic findings and histological characteristics of gastritis. A $P$ value $<0.05$ was considered to indicate statistical significance. Cohen's $\mathrm{k}$ coefficient was then calculated to assess the inter-observer agreement between two endoscopists, and Fleiss's к coefficient was calculated to assess the overall inter-observer agreement among all 6 endoscopists; agreement was regarded as excellent for a $\mathrm{k}$ coefficient of $\geq 0.8$, good for $<0.8$ to $\geq 0.6$, moderate for $<0.6$ to $\geq 0.4$, and fair for $<0.4$. Statistical analyses were performed using $R$ software version 3.1.0 (R Development Core Team, Vienna, Austria).

\section{Results}

\section{Participants}

Among 353 patients who were analyzed in the prospective trial, endoscopic images were not available for 3 and appropriate images were not recorded for another 3 . Consequently, we analyzed 347 lesions (39 cancerous and 308 non-cancerous) ( $>$ Fig. 2). The patient demographics are summarized in $>\mathbf{T a -}$ ble 1. There were 38 differentiated types of cancer and 1 undifferentiated type.

\section{Representative cases}

- Fig. 4a shows a representative lesion with a DL (MCDL, 22/3). As indicated by the arrows, the MCDL was clearly identified as multiple convex-shaped lines along the edge of the epithelia in the surrounding mucosa. The histological diagnosis was gastritis with intestinal metaplasia. $>$ Fig. $\mathbf{4 b}$ shows another representative lesion with a DL $(M C D L,>0$ to $<1 / 3)$. The $D L$ showed
- Table 1 Patient characteristics.

\begin{tabular}{|c|c|c|}
\hline & $\begin{array}{l}\text { Cancer } \\
\mathbf{n}=39\end{array}$ & $\begin{array}{l}\text { Non-cancerous } \\
\text { lesion } \\
\mathrm{n}=\mathbf{3 0 8}\end{array}$ \\
\hline Median age (years) & $72(55-85)$ & $68(37-90)$ \\
\hline \multicolumn{3}{|l|}{ Gender } \\
\hline - Male & 33 & 241 \\
\hline - Female & 6 & 67 \\
\hline \multicolumn{3}{|l|}{ Endoscope } \\
\hline - GIF-Q240Z & 16 & 120 \\
\hline - GIF-H260Z & 21 & 186 \\
\hline - GIF-FQ260Z & 2 & 2 \\
\hline \multicolumn{3}{|l|}{ Location of lesions } \\
\hline - Upper third & 12 & 96 \\
\hline - Middle third & 7 & 72 \\
\hline - Lower third & 20 & 140 \\
\hline \multicolumn{3}{|l|}{ Location of lesions } \\
\hline - Anterior wall & 7 & 53 \\
\hline - Posterior wall & 10 & 89 \\
\hline - Greater curvature & 11 & 63 \\
\hline - Lessor curvature & 11 & 103 \\
\hline Median size of lesions (mm) & $6(3-10)$ & $5(2-10)$ \\
\hline
\end{tabular}

a partly convex shape. The histological diagnosis was differenti-

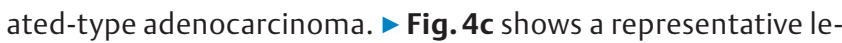
sion without a DL. There was no abrupt change in the microvascular and microsurface patterns between the depressed area and surrounding mucosa. The histological diagnosis was gastritis with intestinal metaplasia.

\section{Diagnostic performance of endoscopic findings for depressed lesions}

The relationship between the presence of a DL and histological findings of the lesions is shown by a flow chart ( $\triangleright$ Fig. 5). A DL was present in 252/347 depressed lesions (73\%), 35/39 cancers (90\%), and 217/308 non-cancerous lesions (70\%). The sensitivity, specificity, PPV, NPV, and accuracy of DL for cancer were $90 \%$ [95\% confidence interval (Cl), $76-97$ ], $30 \%$ (95\% Cl, $25-$ 35), $14 \%(95 \% \mathrm{Cl}, 10-19), 96 \%(95 \% \mathrm{Cl}, 90-99)$, and $36 \%$ (95\% Cl, $31-42)$, respectively.

The relationship between the proportion of an MCDL among lesions with a DL and the histology of the lesions is also shown in $>$ Fig. 5. When the cutoff value for the proportion needed to distinguish non-cancerous lesions from cancer was set at two-thirds, Youden's J index was 0.30 of the maximum value. Therefore, the optimal cutoff value was determined as two-thirds. An MCDL ( $\geq 2 / 3$ ) was present in $86 / 252$ 

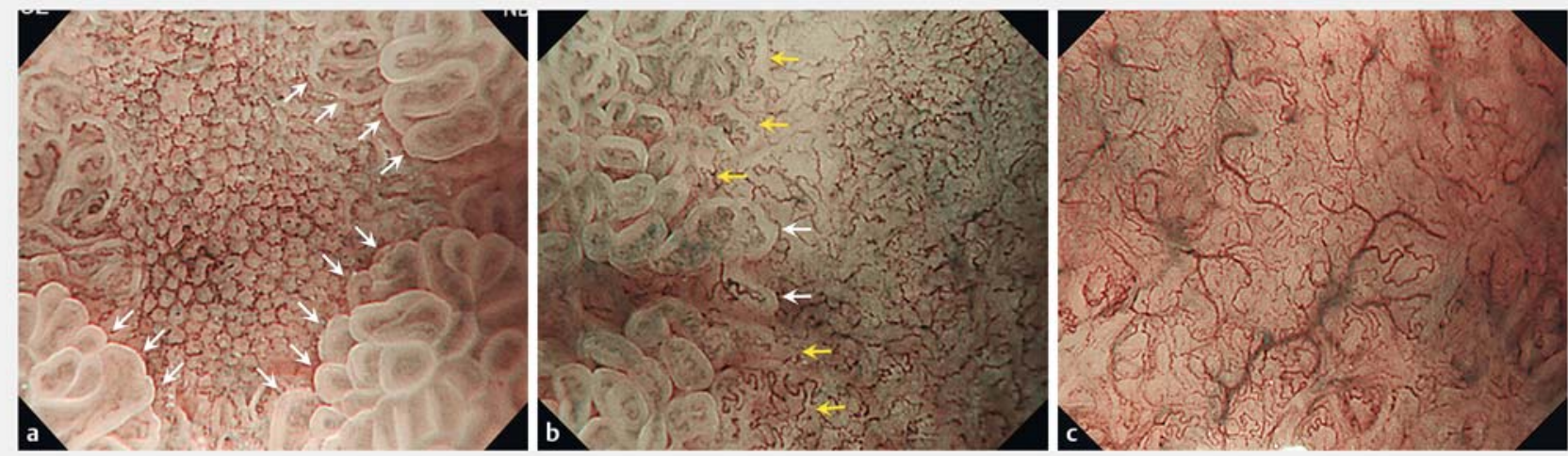

- Fig. 4 a Representative lesion with a DL (MCDL, $\geq 2 / 3$ ). Arrows indicate that the DL was clearly identified as multiple convex-shaped lines along the edge of the epithelia in the surrounding mucosa. The histological diagnosis was gastritis with intestinal metaplasia. $\mathbf{b}$ Representative lesion with a $\mathrm{DL}(\mathrm{MCDL},>0$ to $<1 / 3)$. White arrows indicate that the $\mathrm{DL}$ was identified as convex-shaped lines. Yellow arrows indicate that the $\mathrm{DL}$ was identified as an abrupt change in the microvessel and microsurface pattern of the mucosa but was not identified as multiple convex-shaped lines. The histological diagnosis was differentiated-type adenocarcinoma. c Representative lesion without a DL. There was no abrupt change between the depressed area and the surrounding mucosa. The histological diagnosis was gastritis with intestinal metaplasia. DL, demarcation line; MCDL, multiple convex demarcation line.

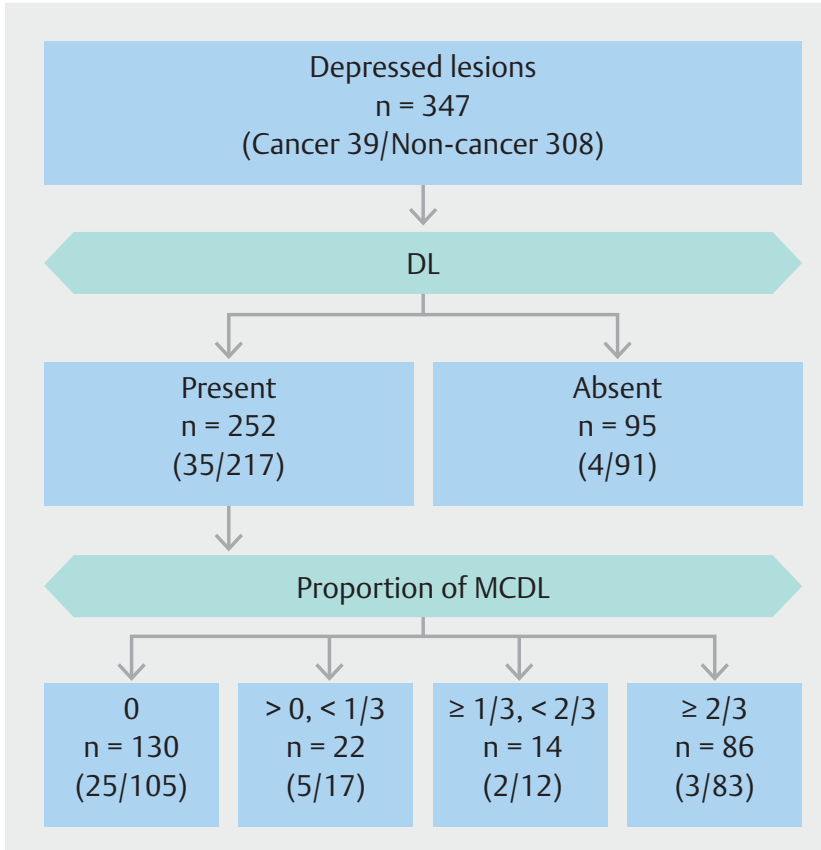

Fig. 5 Relationship between endoscopic findings and histological diagnosis. The number of lesions in each group is indicated in the center tier of the square. The histological diagnosis of each group is placed in parentheses in the lower tier.

depressed lesions (34\%), 3/35 cancers (9\%), and 83/217 noncancerous lesions (38\%). The sensitivity, specificity, PPV, NPV, and accuracy of an MCDL for non-cancerous lesions were $38 \%$ (95\% Cl, $32-45), 91 \%$ (95\% Cl, $77-98), 97 \%$ (95\% Cl, $90-$ 99), $19 \%(95 \% \mathrm{Cl}, 14-26)$, and $46 \%(95 \% \mathrm{Cl}, 39-52)$, respectively.
The diagnostic performances of a DL for cancer and that of a MCDL for non-cancer among the 6 endoscopists are shown in - Table 2 and $>$ Table 3, respectively. Even on average, presence of a DL showed a high sensitivity (93.2\%) and NPV (96.8\%), and presence of an MCDL showed a high specificity (89.3\%) and PPV (96.7\%).

Association of each endoscopic finding with cancer Presence of a DL was associated with diagnosis of cancer (odds ratio, 3.7; $95 \% \mathrm{Cl}, 1.3-14.6 ; P=0.01$ ), and presence of an MCDL showed an inverse association with diagnosis of cancer (odds ratio, $0.15 ; 95 \% \mathrm{Cl}, 0.03-0.51 ; P<0.001$ ).

\section{Lesion localization according to the presence of an MCDL}

Small depressed lesions without an MCDL were most frequently located in the lower third of the stomach, whereas lesions with an MCDL were mainly located in the upper third ( $\vee$ Table 4 ). There was no trend in the circumferential localization of lesions with an MCDL.

\section{Reproducibility of endoscopic findings}

Inter-observer agreement regarding a DL and MCDL between the two experienced endoscopists had a $\mathrm{k}$ coefficient of 0.51 $(95 \% \mathrm{Cl}, 0.42-0.61)$ and $0.70(95 \% \mathrm{Cl}, 0.60-0.79)$, respectively. Inter-observer agreement among all 6 endoscopists ( 2 experienced and 4 less-experienced) for a DL and MCDL had a $\mathrm{k}$ coefficient of 0.36 and 0.54 , respectively. Good agreement for an MCDL was shown between the 2 experienced endoscopists and moderate agreement was shown among all 6 endoscopists. Agreement regarding an MCDL was better than that regarding a DL. 
- Table 2 Diagnostic performance of MCDL for non-cancer.

\begin{tabular}{|l|l|l|l|l|l|}
\hline & Sensitivity & Specificity & PPV & NPV & Accuracy \\
\hline E1 & 38 & 91 & 97 & 19 & 46 \\
\hline E2 & 37 & 91 & 96 & 21 & 46 \\
\hline L1 & 62 & 76 & 95 & 21 & 64 \\
\hline L2 & 15 & 100 & 100 & 15 & 26 \\
\hline L3 & 40 & 78 & 92 & 15 & 46 \\
\hline L4 & 19 & 100 & 100 & 18.2 & 29 \\
\hline Average & 35.2 & 89.3 & 96.7 & 42.8 \\
\hline
\end{tabular}

E, experienced endoscopist; L, less-experienced endoscopist; MCDL, multiple convex demarcation line; NPV, negative predictive value; PPV, positive predictive value.

- Table 3 Diagnostic performance of DL for cancer.

\begin{tabular}{|l|l|l|l|l|l|}
\hline & Sensitivity & Specificity & PPV & NPV & Accuracy \\
\hline E1 & 90 & 30 & 14 & 96 & 36 \\
\hline E2 & 82 & 45 & 16 & 95 & 49 \\
\hline L1 & 97 & 8 & 12 & 96 & 18 \\
\hline L2 & 95 & 17 & 13 & 96 & 25 \\
\hline L3 & 95 & 27 & 14 & 98 & 35 \\
\hline L4 & 100 & 9 & 12 & 100 & 20 \\
\hline Average & 93.2 & 22.7 & 13.5 & 96.8 & 30.5 \\
\hline
\end{tabular}

DL, demarcation line; E, experienced endoscopist; L, less-experienced endoscopist; NPV, negative predictive value; PPV, positive predictive value.

- Table 4 Proportion of lesions with an MCDL in each location.

\begin{tabular}{|c|c|c|c|}
\hline & \multicolumn{3}{|l|}{ MCDL } \\
\hline & $\begin{array}{l}\text { Positive } \\
n=86\end{array}$ & $\begin{array}{l}\text { Negative } \\
n=261\end{array}$ & $P$ value \\
\hline \multicolumn{3}{|c|}{ Longitudinal location (\%) } & $<0.001$ \\
\hline - Upper third & $39(36)$ & $69(64)$ & \\
\hline - Middle third & $22(28)$ & $57(72)$ & \\
\hline - Lower third & $25(16)$ & $135(84)$ & \\
\hline \multicolumn{3}{|c|}{ Circumferential location (\%) } & 0.662 \\
\hline - Anterior wall & $18(30)$ & $42(70)$ & \\
\hline - Posterior wall & $21(21)$ & $78(79)$ & \\
\hline - Greater curvature & $18(24)$ & $56(76)$ & \\
\hline - Lessor curvature & $29(25)$ & $85(75)$ & \\
\hline
\end{tabular}

The $\mathrm{X}^{2}$ test was used. MCDL, multiple convex demarcation line.

\section{Histological characteristics of non-cancerous lesions}

In total, 138 patients with small depressed lesions were enrolled in the prospective study in our institution. Among them, 120 patients were diagnosed with non-cancerous lesions. We excluded 2 patients whose biopsy specimens were lost and evaluated 118 patients to clarify the histological characteristics of gastritis. When the cut-off value for the proportion of MCDLs was set at two-thirds, atrophy and intestinal metaplasia were seen in most of the lesions that were positive for an MCDL ( $97 \%$ and $97 \%$, respectively) ( $\triangleright$ Fig. 6 ). The grade of intestinal metaplasia was significantly higher in the lesions with than without an MCDL $(P=0.028)$. The grades of mononuclear cell infiltration, neutrophil infiltration, and atrophy were not significantly different between these two groups. In contrast, the grade of $\mathrm{H}$. pylori infection was signifiVcantly lower in the lesions with than without an MCDL $(P=0.014)$.

\section{Discussion}

This study demonstrates that the MCDL, which is a novel finding in M-NBI, has a high specificity and PPV for non-cancerous lesions in the differential diagnosis of small depressed lesions. This endoscopic finding may be effective in reducing the number of biopsies from non-cancerous lesions. Predicting non- 


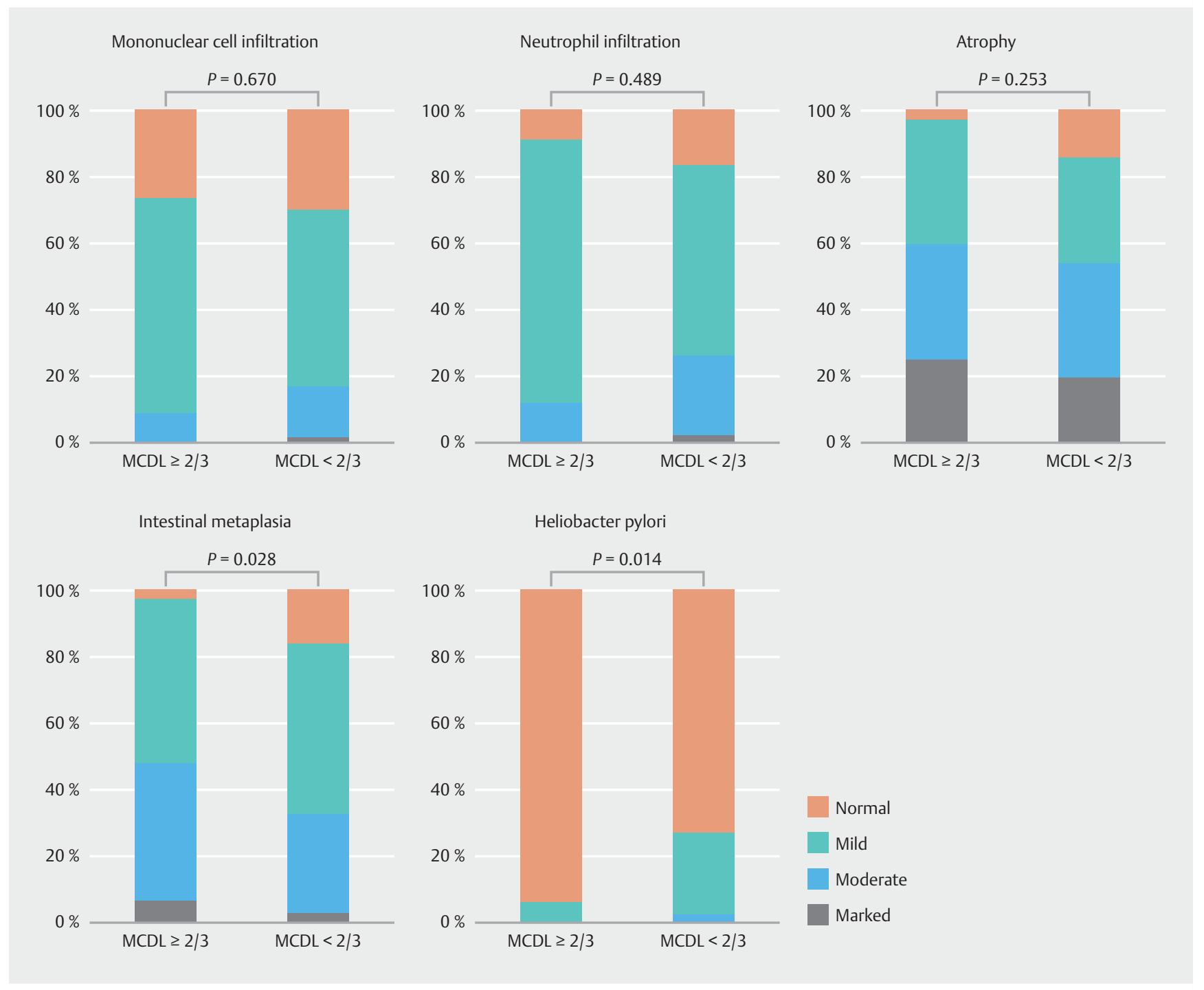

- Fig. 6 Association between MCDL comprising more than two-thirds of DL and the grade of gastritis. DL, demarcation line; MCDL, multiple convex demarcation line.

cancerous lesions is significant and practical because our study showed that benign depressed lesions were detected 7.8 times as frequently as cancer in screening endoscopy in high-risk patients [5].

In VS classification system and Magnifying Endoscopy Simple Diagnostic Algorithm for Early Gastric Cancer (MESDA-G), evaluation for presence of a DL is recommended first, followed by the evaluation of irregular microvascular and/or microsurface patterns in lesions with a DL $[19,20]$. The strategy is based on the high sensitivity and low specificity of a DL for early gastric cancer [19]. Non-cancerous lesions occasionally show a DL, because the microsurface structure exhibits a mosaic pattern in atrophic gastritis [21]. According to the present results, we consider that an MCDL is a predictor of non-cancerous lesions. The diagnostic strategy for DL evaluation is indicated in > Fig.7. When the cut-off value for the proportion of MCDLs was set at two-thirds, an MCDL was observed in $25 \%$ (86/347) of small depressed lesions detected by screening endoscopy. Participants in this study were patients at high risk of gastric cancer; i. e., patients with gastric cancer or a history of endoscopic resection of gastric cancer. The annual incidence rate for metachronous multiple cancers in patients with gastric cancer is higher than that for newly gastric cancer in the general populations; the former was $3.5 \%$ and the latter was $0.016-1.1 \%[22,23]$. In the current study, we analyzed 347 (98\%) of 353 patients who were prospectively enrolled in 9 hospitals. Therefore, the selection bias of our data is estimated to be low, and we believe that the incidence of an MCDL reflects the actual number of patients at high risk of gastric cancer in this study.

Diagnostic performance of a DL had high sensitivity (90\%) but low specificity (30\%), and the PPV was $14 \%$; this means that $86 \%$ of the lesions with a DL were non-cancerous lesions. According to the MESDA-G [20], evaluation for an IMVP is recommended for lesions with a DL. Although presence of an IMVP had a high diagnostic value for cancer, endoscopists' judgments are subjective, and considerable experience is re- 


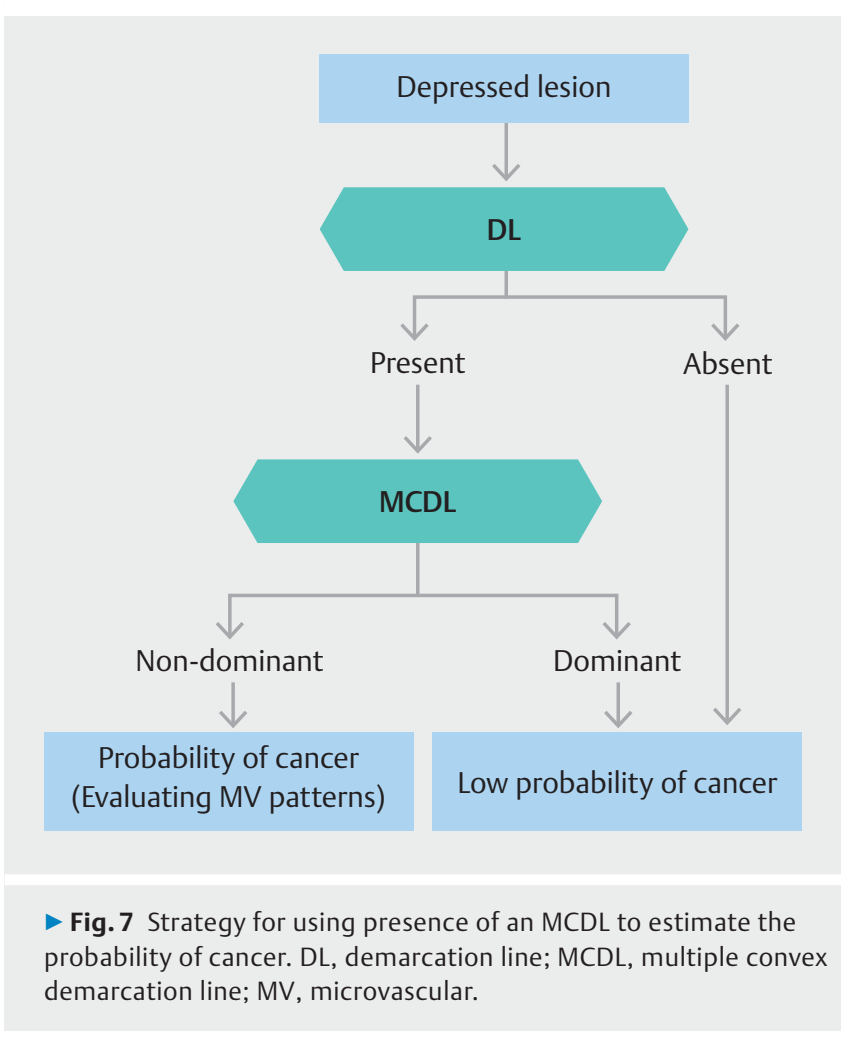

quired for accurate diagnosis using M-NBI. Yoo et al. [13] reported that the inter-observer agreement regarding the IMVP between 2 expert endoscopists was moderate ( $\mathrm{k}$ value of 0.541 ). We also assessed the inter-observer agreement regarding the IMVP in our study, and the $\mathrm{K}$ value was 0.42 between the 2 experienced endoscopists and 0.30 among all 6 endoscopists (supplementary data). Inter-observer agreement for the MCDL was higher than that for the IMVP. Presence of an MCDL can exclude non-cancerous lesions with high specificity and good inter-observer variability compared with evaluation of the microvascular architecture.

Why is an MCDL specifically found in non-cancerous lesions? Identification of an MCDL requires clear visualization of the marginal crypt epithelium (MCE) of the surrounding mucosa at the boundary of the lesion ( $\triangleright$ Fig. 8a). In M-NBI, clear visualization of the MCE reflects the perpendicular arrangement of the epithelial cells [24]. An MCDL is detected by M-NBI in the depressed areas that meet such histological conditions. Yagi et al. [25] reported that the shallow depth of the crypts and the short length of their intervening parts are associated with disappearance of the MCE. We speculate that these histological changes occur at the invasion front of cancer. Intraepithelial microinvasion, which is often seen in early gastric cancer, disturbs the perpendicular arrangement of epithelial cells in the surrounding mucosa [26], and the crypts of the surrounding mucosa become shallower $>$ Fig. 8 b).

In the current study, histological investigation of the noncancerous lesions suggested that the presence of an MCDL $(\geq 2 / 3)$ was associated with a higher grade of intestinal metaplasia than other proportions of an MCDL. Although there was a significant difference, even the latter was frequently associat- ed with intestinal metaplasia. The biopsy specimens were obtained according to the protocol in the prospective trial. Most of the target lesions were smaller (median size of $5 \mathrm{~mm}$ ) than the opening width of the biopsy forceps; therefore, the specimens may have been contaminated with the surrounding mucosa that contained intestinal metaplasia. Moreover, "groovetype" mucosa, which was proposed by Kanzaki et al. [21] as an $\mathrm{M}-\mathrm{NBI}$ finding associated with atrophy and intestinal metaplasia, was more frequently present in the surrounding mucosa of lesions with an MCDL $\geq 2 / 3$ than in other lesions ( Supplementary Table 1). In the present study, we evaluated the status of $\mathrm{H}$. pylori infection by histological examination. However, the information regarding eradication therapy for $\mathrm{H}$. pylori infection was quite limited: Of 94 patients without $\mathrm{H}$. pylori infection, 30 (32\%) had undergone eradication therapy, 8 (9\%) had not undergone eradication therapy, and 56 (60\%) had unclear data. Among the 64 patients with an absent or unclear eradication history, histological evaluation showed atrophy was in 55 (86\%) patients. Patients with no $\mathrm{H}$. pylori infection were considered rare among the participants of this study.

Our study had some limitations. First, we evaluated the endoscopic findings retrospectively; therefore, some DLs might not have been recorded in the images and evaluated. However, we do not believe that this greatly affected our results because the target lesions were small. Second, the number of cancerous lesions was small. In particular, only one case of undifferentiated cancer was included. Therefore, whether our findings are applicable to undifferentiated cancer is not clear. Third, only patients at high risk for gastric cancer were enrolled, which makes the diagnostic yield of MCDL in the general population unclear. Fourth, non-depressed lesions were not considered in the present study, and the size of the target lesions was limited to $\leq 10 \mathrm{~mm}$; therefore, whether this finding is applicable to nondepressed lesions or larger lesions remains unclear. However, the MCDL is considered to be meaningless for characterization of elevated lesions because we observed no MCDLs in elevated lesions. Finally, the cutoff value of the MCDL and results of the present study have not yet been sufficiently verified yet. A prospective study is expected for validation.

\section{Conclusion}

In summary, the MCDL observed in M-NBI has a high specificity and PPV for non-cancerous depressed lesions and high interobserver agreement. the presence of an MCDL is considered to be associated with intestinal metaplasia and the absence of $H$. pylori. Evaluating the shape of the DL might be a useful method for differential diagnosis between early gastric cancer and non-cancerous depressed lesions.

\section{Acknowledgements}

We thank Angela Morben, DVM, ELS, from Edanz Group (www. edanzediting.com/ac) for editing a draft of this manuscript. 


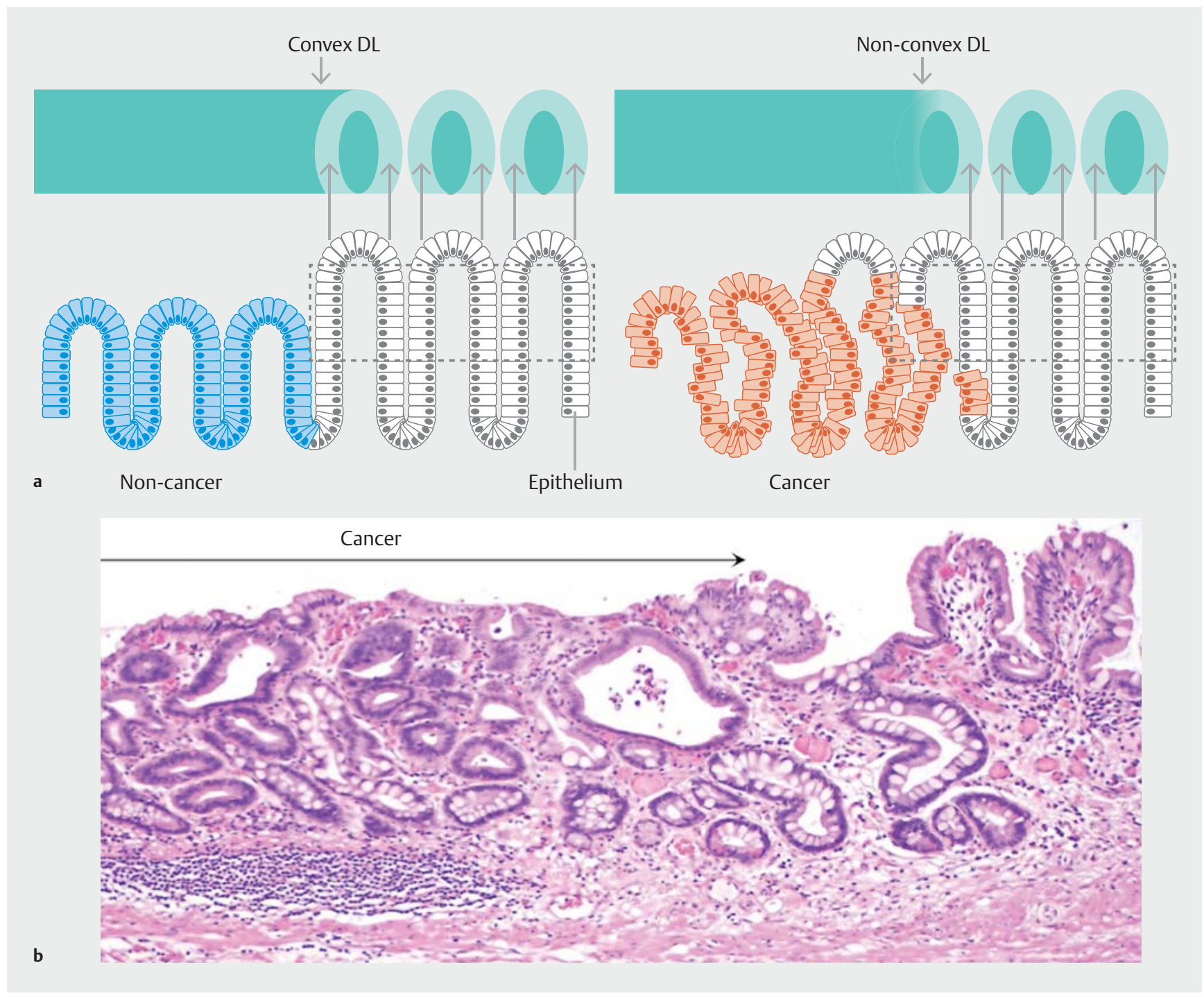

- Fig. 8 a Graphic representation of a convex DL (left side) and non-convex DL (right side). The upper row shows M-NBI images and the lower row, histological structures. As indicated by the boxes and arrows, the MCE in M-NBI reflects the perpendicular arrangement of the epithelial cells. When the MCE appears at the boundary of the depressed lesion, the DL shows a clear convex shape. Cancer disturbs the perpendicular arrangement of epithelial cells in the surrounding mucosa. Therefore, the MCE does not appear at the boundary of the depressed lesion. b Histological image of cancer. DL, demarcation line; M-NBI, magnifying narrow-band imaging; MCE, marginal crypt epithelium.

\section{Competing interests}

None

References

[1] Allum WH, Blazeby JM, Griffin SM et al. Guidelines for the management of oesophageal and gastric cancer. Gut 2011; 60: 1449-1472

[2] Thrumurthy SG, Chaudry MA, Hochhauser D et al. The diagnosis and management of gastric cancer. BMJ 2013; 347: f6367

[3] Gotoda T, Yanagisawa A, Sasako M et al. Incidence of lymph node metastasis from early gastric cancer: estimation with a large number of cases at two large centers. Gastric Cancer 2000; 3: 219-225

[4] Everett SM, Axon AT. Early gastric cancer in Europe. Gut 1997; 41: $142-150$
[5] Ezoe $\mathrm{Y}$, Muto $\mathrm{M}$, Uedo $\mathrm{N}$ et al. Magnifying narrowband imaging is more accurate than conventional white-light imaging in diagnosis of gastric mucosal cancer. Gastroenterology 2011; 141: 2017-2025

[6] Gono K, Obi T, Yamaguchi M et al. Appearance of enhanced tissue features in narrow-band endoscopic imaging. J Biomed Opt 2004; 9: $568-577$

[7] Muto M, Katada C, Sano Y et al. Narrow band imaging: a new diagnostic approach to visualize angiogenesis in the superficial neoplasia. Clin Gastroenterol Hepatol 2005; 3: S16 - 20

[8] Yao K, Anagnostopoulos GK, Ragunath K. Magnifying endoscopy for diagnosing and delineating early gastric cancer. Endoscopy 2009; 41 : $462-467$

[9] Yao K, Oishi T, Matsui T et al. Novel magnified endoscopic findings of microvascular architecture in intramucosal gastric cancer. Gastrointest Endosc 2002; 56: 279-284

[10] Yao K, Iwashita A, Kikuchi Y et al. Novel zoom endoscopy technique for visualizing the microvascular architecture in gastric mucosa. Clin Gastroenterol Hepatol 2005; 3: S23-26 
[11] Yao K, Iwashita A, Tanabe H et al. Novel zoom endoscopy technique for diagnosis of small flat gastric cancer: a prospective, blind study. Clin Gastroenterol Hepatol 2007; 5: 869-878

[12] Yao K, Doyama H, Gotoda T et al. Diagnostic performance and limitations of magnifying narrow-band imaging in screening endoscopy of early gastric cancer: a prospective multicenter feasibility study. Gastric Cancer 2014; 17: 669-679

[13] Yoo CH, Park MI, Park S] et al. Observer variability in gastric neoplasm assessment using the vessel plus surface classification for magnifying endoscopy with narrow band imaging. Clin Endosc 2014; 47: $74-78$

[14] Bossuyt PM, Reitsma JB, Bruns DE et al. The STARD statement for reporting studies of diagnostic accuracy: explanation and elaboration. Ann Intern Med 2003; 138: W1 - 12

[15] Kanesaka T, Uedo N, Yao K et al. A significant feature of microvessels in magnifying narrow-band imaging for diagnosis of early gastric cancer. Endosc Int Open 2015; 3: E590 - 596

[16] Schlemper RJ, Riddell RH, Kato Y et al. The Vienna classification of gastrointestinal epithelial neoplasia. Gut 2000; 47: 251-255

[17] Yao K. Zoom gastroscopy: Magnifying endoscopy in the stomach. Tokyo: Springer; 2014: 12.4: $91-92$

[18] Dixon MF, Genta RM, Yardley JH et al. Classification and grading of gastritis. The updated Sydney System. International Workshop on the Histopathology of Gastritis, Houston 1994. Am J Surg Pathol 1996; 20: $1161-1181$

[19] Yamada S, Doyama H, Yao K et al. An efficient diagnostic strategy for small, depressed early gastric cancer with magnifying narrow-band imaging: a post-hoc analysis of a prospective randomized controlled trial. Gastrointest Endosc 2014; 79: 55-63

[20] Muto M, Yao K, Kaise M et al. Magnifying endoscopy simple diagnostic algorithm for early gastric cancer (MESDA-G). Dig Endosc 2016; 28: 379-393

[21] Kanzaki H, Uedo N, Ishihara R et al. Comprehensive investigation of areae gastricae pattern in gastric corpus using magnifying narrow band imaging endoscopy in patients with chronic atrophic fundic gastritis. Helicobacter 2012; 17: 224-231

[22] Kato M, Nishida T, Yamamoto K et al. Scheduled endoscopic surveillance controls secondary cancer after curative endoscopic resection for early gastric cancer: a multicenter retrospective cohort study by Osaka University ESD study group. Gut 2013; 62: 1425-32

[23] Yoshida T, Kato J, Inoue I et al. Cancer development based on chronic active gastritis and resulting gastric atrophy as assessed by serum levels of pepsinogen and Helicobacter pylori antibody titer. Int J Cancer 2014; 134: 1445 - 1457

[24] Yao K. Zoom gastroscopy: Magnifying endoscopy in the stomach. Tokyo: Springer; 2014: 8.3: 61-69

[25] Yagi K, Nozawa Y, Endou S et al. Diagnosis of early gastric cancer by magnifying endoscopy with NBI from viewpoint of histological imaging: mucosal patterning in terms of white zone visibility and its relationship to histology. Diagn Ther Endosc 2012; 2012: 954809

[26] Yao K. Zoom gastroscopy: Magnifying endoscopy in the stomach. Tokyo: Springer; 2014: 13.1: 100-125 
- Supplementary Table 1 Association of mucosal pattern of surrounding mucosa between lesions with and without an MCDL.

\begin{tabular}{|l|c|c|c|}
\hline & MCDL $\geq 2 / 3$ & MCDL $<1 / 3$ & P value \\
\hline Groove type & $68(79)$ & $170(64)$ & 0.014 \\
\hline Mixed type & $10(12)$ & $29(11)$ & \\
Foveola type & $8(9)$ & $62(24)$ & \\
\hline
\end{tabular}

MCDL, multiple convex demarcation line. The $x^{2}$ test was used. 